\title{
Correction
}

\section{Correction: Ten et al., Complement Component C1q Mediates Mitochondria-Driven Oxidative Stress in Neonatal Hypoxic-Ischemic Brain Injury}

In the article "Complement Component C1q Mediates Mitochondria-Driven Oxidative Stress in Neonatal Hypoxic-Ischemic Brain Injury” by Vadim S. Ten, Jun Yao, Veniamin Ratner, Sergey Sosunov, Deborah A. Fraser, Marina Botto, Baalasubramanian Sivasankar, B. Paul Morgan, Samuel Silverstein, Raymond Stark, Richard Polin, Susan J. Vannucci, David Pinsky, and Anatoly A. Starkoy, which appeared on pages 2077-2087 of the February 10, 2010 issue, the authors regret an error in Figure 1A and E. The same image of a $\mathrm{Clq}^{-1-}$ mouse contralateral hemisphere appeared in both panels. A corrected figure appears below. The interpretation and conclusions of the study are not affected by this error.

\section{$\mathrm{C} 3 \mathrm{~b} / \mathrm{iC} 3 \mathrm{~b} / \mathrm{C} 3 \mathrm{c}$}
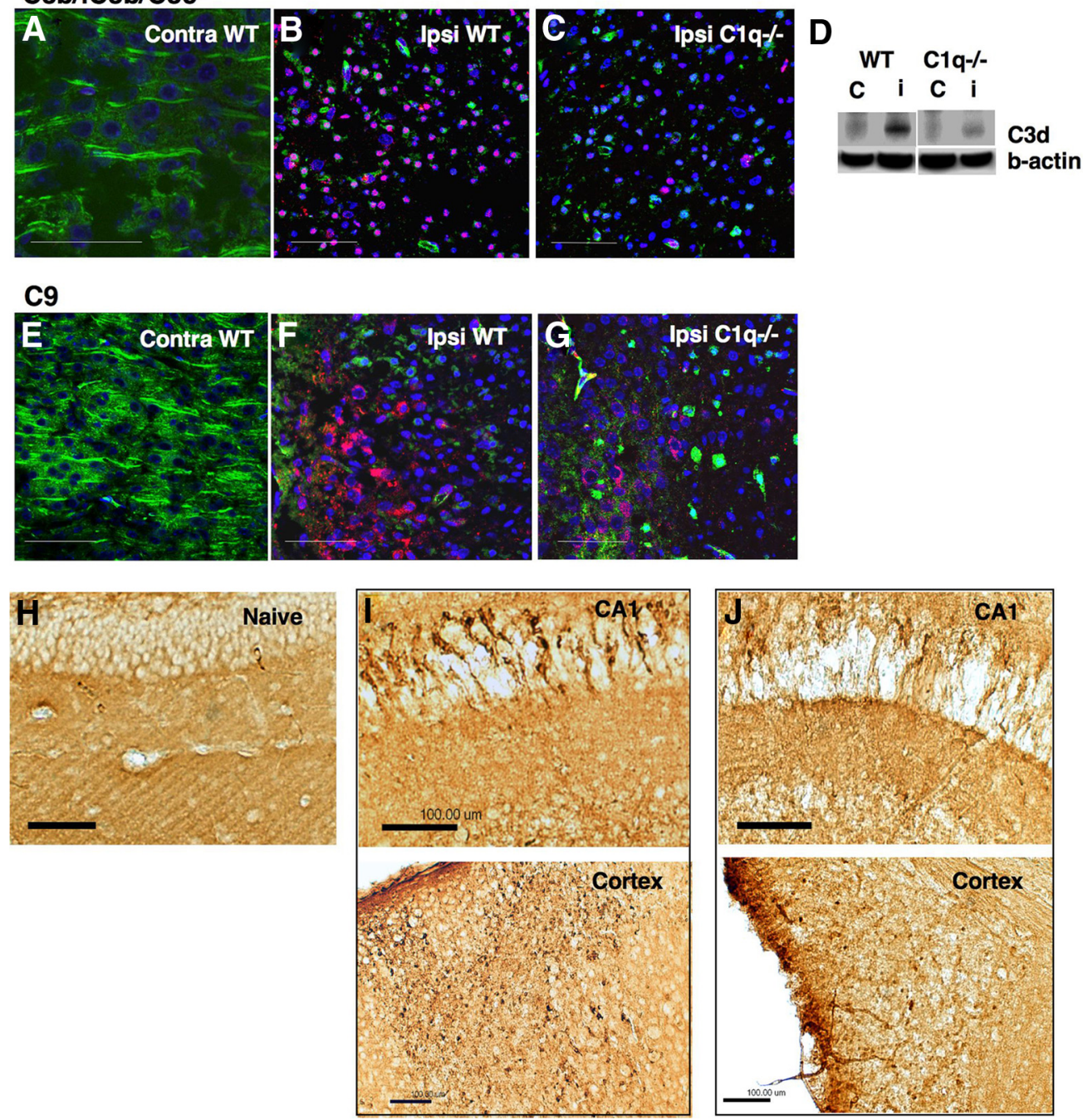

Figure 1.

DOI: 10.1523/JNEUROSCI.4844-13.2014 